\title{
mTOR signaling pathway and mTOR inhibitors in cancer: progress and challenges
}

\author{
Zhilin Zou ${ }^{1,2,3 \dagger}, \mathrm{Tao} \mathrm{Tao}^{4 \dagger}$, Hongmei $\mathrm{Li}^{3^{*}}$ and Xiao Zhu ${ }^{1,2^{*}}$ (1)
}

\begin{abstract}
Mammalian target of rapamycin (mTOR) regulates cell proliferation, autophagy, and apoptosis by participating in multiple signaling pathways in the body. Studies have shown that the mTOR signaling pathway is also associated with cancer, arthritis, insulin resistance, osteoporosis, and other diseases. The mTOR signaling pathway, which is often activated in tumors, not only regulates gene transcription and protein synthesis to regulate cell proliferation and immune cell differentiation but also plays an important role in tumor metabolism. Therefore, the mTOR signaling pathway is a hot target in anti-tumor therapy research. In recent years, a variety of newly discovered mTOR inhibitors have entered clinical studies, and a variety of drugs have been proven to have high activity in combination with mTOR inhibitors. The purpose of this review is to introduce the role of mTOR signaling pathway on apoptosis, autophagy, growth, and metabolism of tumor cells, and to introduce the research progress of mTOR inhibitors in the tumor field.
\end{abstract}

Keywords: mTOR signaling pathway, mTOR inhibitor, Tumor metabolism, Autophagy, Apoptosis

\section{Background}

The mTOR forms two structurally and functionally distinct complexes called the mammalian target of rapamycin complex 1 (mTORC1) and mammalian target of rapamycin complex 2 (mTORC2). mTORC1 is comprised of mTOR, raptor, $\mathrm{G} \beta \mathrm{L}$ and deptor, while mTORC2 is composed of mTOR, Rictor, GßL, PRR5, deptor, and SIN1. mTORC1 integrates signals from multiple growth factors, nutrients, and energy supply to promote cell growth when energy is sufficient and catabolism when the body is hungry. mTORC1 mainly regulates cell growth and metabolism, while mTORC2 mainly controls cell proliferation and survival [1]. Studies have shown that mTOR is involved in many signaling pathways in the body, including phosphoinositide-3-kinase (PI3K)/AKT,

\footnotetext{
*Correspondence: lihongmei@gdmu.edu.cn; xzhu@gdmu.edu.cn

${ }^{\dagger}$ Zhilin Zou and Tao Tao contributed equally to this work

1 Guangdong Key Laboratory for Research and Development of Natural Drugs, Guangdong Medical University, Zhanjiang, China

${ }^{3}$ Department of Pathology, Guangdong Medical University, Dongguan, China

Full list of author information is available at the end of the article
}

tuberous sclerosis complex subunit 1 (TSC1)/tuberous sclerosis complex subunit 2 (TSC2)/Rheb, LKBL/ adenosine $5^{\prime}$-monophosphate-activated protein kinase (AMPK), VAM6/Rag GTPases and so on [2]. It influences transcription and protein synthesis by integrating various signal stimulation, and finally regulates apoptosis, growth, and autophagy of cells [3]. Scientists have also linked mTOR to various disease processes, such as tumor formation, arthritis, insulin resistance and osteoporosis $[4,5]$. Among them, mTOR plays a key role in tumor tumorigenesis and development. And multiple studies have suggested that tumors typically over-activate the AKT/mTOR signaling pathway [6, 7]. Therefore, mTOR inhibitors are widely used in the research of targeted therapy for tumors, organ transplantation, rheumatoid arthritis, and other diseases. The purpose of this review is to elucidate the relationship between mTOR signaling pathway and tumor development and to introduce the research progress of mTOR inhibitors.

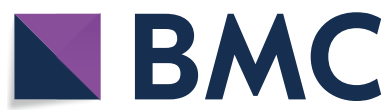

(c) The Author(s) 2020. This article is licensed under a Creative Commons Attribution 4.0 International License, which permits use, sharing, adaptation, distribution and reproduction in any medium or format, as long as you give appropriate credit to the original author(s) and the source, provide a link to the Creative Commons licence, and indicate if changes were made. The images or other third party material in this article are included in the article's Creative Commons licence, unless indicated otherwise in a credit line to the material. If material is not included in the article's Creative Commons licence and your intended use is not permitted by statutory regulation or exceeds the permitted use, you will need to obtain permission directly from the copyright holder. To view a copy of this licence, visit http://creativeco mmons.org/licenses/by/4.0/. The Creative Commons Public Domain Dedication waiver (http://creativecommons.org/publicdomain/ zero/1.0/) applies to the data made available in this article, unless otherwise stated in a credit line to the data. 


\section{The relationship between mTOR signaling pathway and tumors}

\section{Tumor growth and proliferation}

Under normal circumstances, mTOR is a major regulator of cell growth and division. However, in tumor cells, abnormally activated mTOR sends signals that encourage tumor cells to grow, metastasize, and invade new healthy tissues [8]. Among them, PI3K/phosphate and fungi homology deleted on chromosome 10 (PTEN)/AKT/ TSC pathway is the main activator of mTORC1, and gene mutations in this pathway can lead to malignant tumors [9]. In addition, the expression of PTEN is often eliminated by epigenetic, genetic, and post-transcriptional modification to up-regulate the PI3K/Akt/mTOR pathway in most malignant tumors [10].

Hou et al. [11] found that mutations in the PTEN gene led to abnormal activation of the PI3K/PTEN pathway in hepatic cell carcinoma (HCC). Furthermore, deletion of the PTEN gene induces the expression of B7-H1, which leads to immunosuppression and increases tumor progression and invasion [12]. In liver cancer, the PI3K/ PTEN/Akt/mTOR pathway activated is involved in tumor invasion and metastasis by up-regulating matrix metallopeptidase 9 (MMP-9) [13]. Similarly, the PI3K/ Akt/mTOR signaling pathway has been found to control the proliferation and survival of colon cancer stem cells (CCSC). In sporadic colon cancer, CCSC may cause recurrence and metastasis [14]. Xie et al. [15] found that liver kinase B1 (LKB1) gene mutation or extracellular growth signal could activate mTORC1. MTORC1 inhibits the activity of ring finger protein 168 (RNF168) protein and promotes its degradation by phosphorylating the 60th serine of RNF168. This will significantly reduce the ubiquitination modification of histone $\mathrm{H} 2 \mathrm{~A}$ and $\mathrm{H} 2 \mathrm{~A}$ histone family member X (H2AX) after DNA damage, which will inhibit the response to DNA damage and reduce the stability of the genome, leading to the promotion of malignant cell transformation and cancer. In addition, existing research shows that Rheb is a GTPase that binds and activates mTORC1 when GTP is loaded. Deng et al. [16] reported that the ubiquitination of Rheb was regulated by growth factor signals. Ubiquitinated Rheb inhibits Rheb activity by promoting Rheb binding to TSC2, leading to the inhibition of mTORC1 expression. In addition to the mTORC1 pathway, the mTORC2 pathway is also involved in the regulation of the occurrence and development of tumor cells. Wang et al. [17] demonstrated that OTU deubiquitinase 7B (OTUD7B) reduced ubiquitination level of $G \beta L$ to prevent $G \beta L$ from interacting with SIN1, leading to activation of mTORC2/ AKT signaling pathway and down-regulation of mTORC1 expression. This partially activates AKT oncogenic signaling and promotes tumorigenesis. However, the ubiquitin ligase TNF Receptor Associated Factor 2 (TRAF2) has the opposite effect by increasing the level of GßL ubiquitination. Similarly, Kovalski et al. [18] proved that Ras mutations can bind to mTOR of mTORC2 and mitogen-activated protein kinase-associated protein 1 (MAPKAP1) to promote the activity of mTORC2 kinase, thus initiating downstream proliferative cell cycle transcription programs.

In summary, mTOR is always stimulated in tumors to maintain the growth, survival and proliferation of tumor cells, and plays a key role in tumor cell biology (Fig. 1).

\section{Tumor metabolism}

mTOR is activated when nutrients are sufficient, which promotes anabolism and energy storage and utilization. When nutrients are relatively scarce, the body must inhibit the activation of mTOR to keep cell material and energy stable. Tumor cells require large amounts of proteins, lipids, and nucleotides to respond to their needs for growth and division [19]. Therefore, abnormal activity of the mTOR pathway often occurs in tumors, because mTOR plays a core role in regulating metabolism.

In breast cancer cells, the PI3K/AKT/mTORC1/sterol regulatory element-binding protein (SREBP) pathway is the main mechanism to induce new lipid synthesis and promote tumor proliferation [20]. Pyruvate kinase (PK) is involved in sugar metabolism while fatty acid synthase (FASN) is involved in the synthesis of fatty acid (FA). Tao et al. [21] found that down-regulating the expression of pyruvate kinase M2 (PKM2) deactivates the AKT/mTOR signaling pathway, thereby reducing the expression of SREBP-1c. The reduced expression level of SREBP-1c inhibits the generation of FA by inhibiting the transcription of the FASN gene, resulting in the inhibited growth of tumor cells. In addition, Di Malta et al. [22] reported that the up-regulated transcription factor enhancer (TFE) gene can activate the Rag GTPase/mTORC1 pathway. In normal cells, this pathway is activated so that cells can better absorb nutrients to maintain physiological functions. In tumor cells, this pathway is often over-activated to meet the nutritional needs of the rapidly growing tumor cells. However, Guri et al. [23] explored that mTORC2 promoted the production of sphingomyelin and cardiolipin in HCC. On the one hand, sphingomyelin and cardiac phospholipids are both structural components of cell biofilms. On the other hand, the metabolism and transport of cardiac phospholipids contribute to the proper functioning of mitochondria, so they must be supplied in large quantities in rapidly proliferating tumor cells [24]. These results suggest that the mTORC2 signaling pathway promotes HCC proliferation and energyrelated lipid production. 


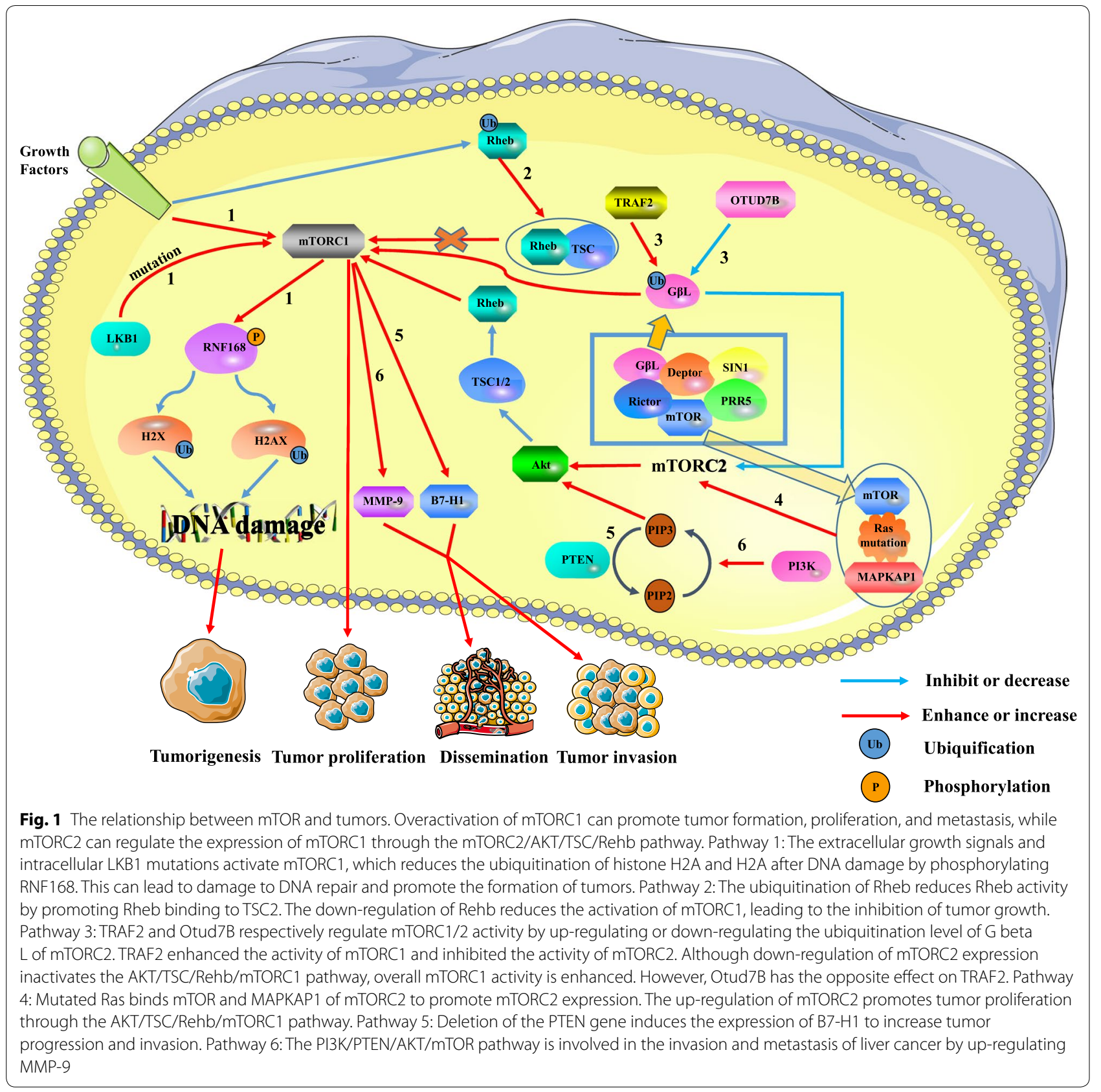

As mentioned above, the metabolisms of tumor cells can be regulated by the mTOR pathway to meet their proliferative and nutritional needs. Conversely, tumor cell metabolism can also promote tumor growth through the mTOR pathway. Ericksen et al. [25] demonstrated that the reduction of branched-chain amino acid (BCAA) decomposition could promote the occurrence and development of tumors by enhancing the activity of mTORC1. They also concluded that the activity of the key enzyme in the BCAA catabolism process was highly correlated with tumor invasion. Therefore, BCAAs accumulation caused by inhibition of BCAA catabolism in liver tumor tissues may be the primary mechanism of chronic activation of tumor mTORC1. Similarly, Shi et al. [26] showed that the expression of adenosine A2a receptor (A2aR) in gastric cancer (GC) tissues was increased, and the expression of $A 2 a R$ was positively correlated with the GC stage. The results suggest that adenosine activates the PI3K/AKT/mTOR signaling pathway by binding to A2aR, which ultimately 
promotes the progress of GC. Madak-Erdogan et al. [27] found that free fatty acids (FFAs) activated estrogen receptor $\alpha(E R \alpha)$ and mTOR pathways, which were correlated with higher proliferation and invasiveness of ER (+) breast cancer cells.

These studies indicate that the mTOR signaling pathway is closely related to tumor metabolism, and provide theoretical support for the combined application of mTOR inhibitors and some drugs that interfere with tumor metabolism (Fig. 2).

\section{Immune cells}

Tumors can develop immune tolerance by suppressing the immune system's ability to recognize and kill tumor cells. Tumor immunotherapy is a hot research topic in recent years [28], and a variety of evidence shows that mTOR pathway, which is often abnormally activated in tumors, can regulate the differentiation and function of immune cells.

\section{$T$ cells}

T-progenitor cells from the bone marrow enter the thymus, where they differentiate into two types of cells: $\alpha \beta$ $\mathrm{T}$ cells and $\gamma \delta$ T cells. Through the gene knockout experiment, Yang et al. [29] proved that RAPTOR deletion in mTORC1 would break the remodeling process of oxidative metabolism and glucose metabolism during $\mathrm{T}$ cell differentiation. This triggers the production of reactive oxygen species (ROS), which disrupts the differentiation of $\alpha \beta \mathrm{T}$ cells and increase the differentiation of $\gamma \delta \mathrm{T}$ cells. In addition, Essig et al. [30] showed that roquin could down-regulate the expression of the PI3K/mTOR pathway. The downregulation of the PI3K/mTOR pathway not only inhibits the differentiation and activation of traditional $\mathrm{T}$ cells, but also limits the specialization of Treg cells. Pollizzi et al. [31] found in mouse models that activation of mTORC1 promoted the production of a cluster of differentiation (CD) $8+$ effector T cells with high glycolysis. These $\mathrm{T}$ cells retain the effector phenotype but do not convert to a memory phenotype. In contrast, $\mathrm{T}$ cells

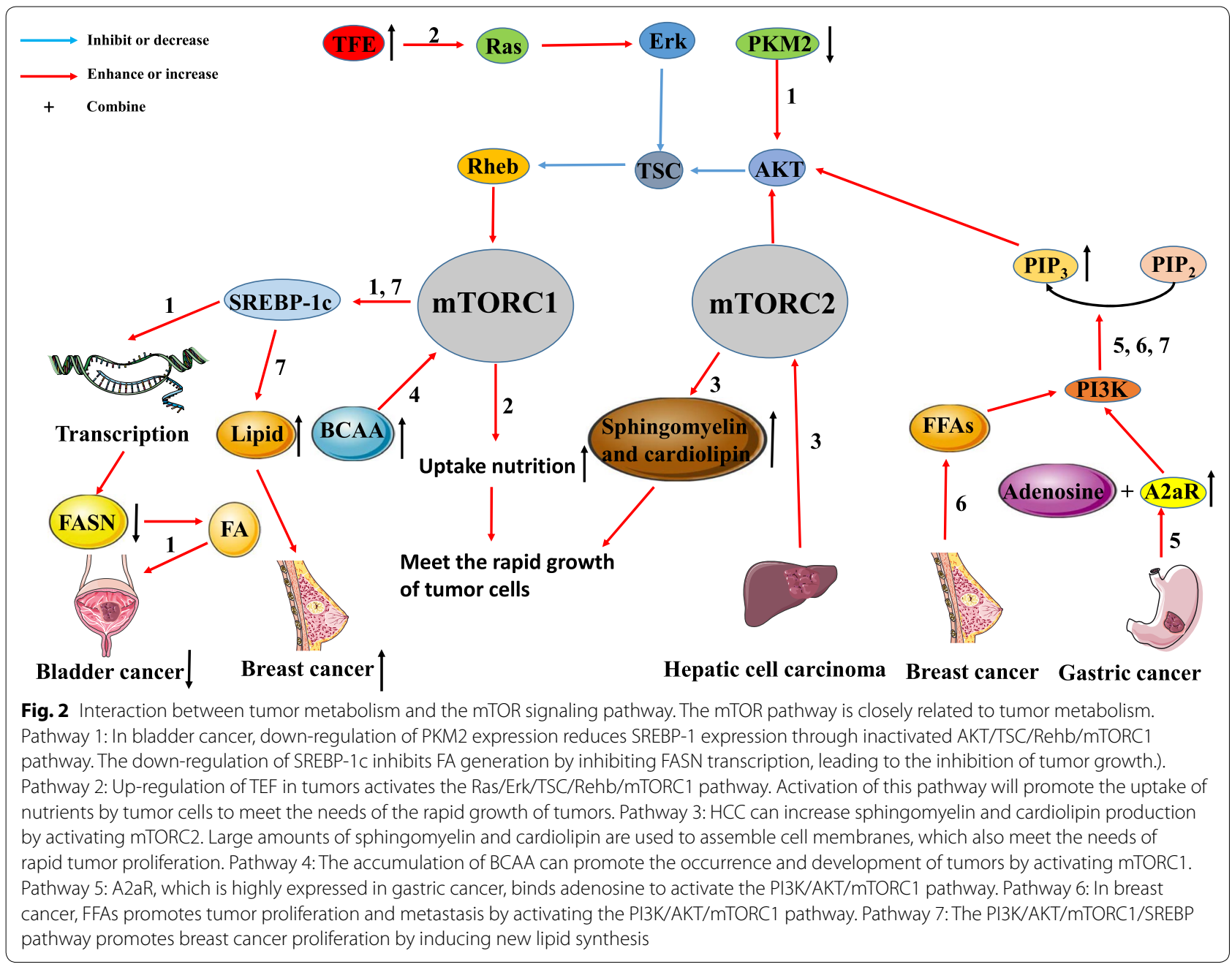


with reduced activity of mTORC1 have the characteristics of memory cells but cannot differentiate into effector cells, and they cannot produce an immune memory response due to the defective metabolic function. Further studies showed that mTORC1 could affect the effector function of $\mathrm{CD} 8+\mathrm{T}$ cells, while mTORC2 activity regulated the memory ability of CD8+ $\mathrm{T}$ cells.

In general, the mTOR signaling pathway can affect the differentiation and function of $\mathrm{T}$ cells. These studies extend our current understanding of $\mathrm{T}$ cell biology.

\section{Natural killer cells and dendritic cells}

The mTOR signaling pathway not only regulates $\mathrm{T}$ cells, but is closely related to the differentiation and functions of dendritic cells (DCs) and natural killer (NK) cells. DC has a strong antigen presentation ability, and NK cells are important immune cells in the body. Wang et al. [32] proved that mTORC1 and mTORC2 regulate NK cell effects in a unique way. They found that mTORC2 negatively regulates NK cell function mainly by inhibiting the signal transducer and activator of transcription 5 (STAT5)/solute carrier family 7 member 5 (SLC7A5) axis. While mTORC1 positively regulates the activity of mTORC2 by maintaining the CD122-mediated interleukin (IL)-15 signaling pathway. In addition to NK cells, DC is also believed to be related to the mTOR signaling pathway in recent years. Chen et al. [33] concluded that apoptosis of DC-derived from bone marrow mononuclear (BMM) cells was reduced after treatment with mTOR inhibitors. They also found that BMM cellderived DCs had better antigen presentation capabilities and that e7-specific cytotoxic CD8+ T lymphocytes activated by these DCs had greater anti-tumor activity. Therefore, mTOR inhibitors can enhance the efficacy of tumor immunotherapy by extending the life span of DC, improving antigen presentation and antigen processing ability. These studies provide ideas for targeting NK and DC cells for anti-tumor therapy.

\section{Macrophages}

M1-type macrophages can kill tumor cells in multiple ways, while M2-type macrophages promote the occurrence, invasion, and metastasis of the tumors. Zhihua et al. [34] reported that the expression of microRNA (miRNA)-30c was significantly reduced in GC. Further studies showed that hypoxia-inducible factor- $1 \alpha$ (HIF- $1 \alpha)$ inhibited miRNA-30c expression. The downregulation of miRNA-30c will reduce mTOR activity and glycolysis in tumor-related macrophages. This will eventually promote GC growth and metastasis by inhibiting the differentiation and function of M1-type macrophages.

In general, the mTOR pathway, which is often activated in tumors, promotes tumor growth by regulating the differentiation and function of immune cells. This will play a positive role in the exploration of new immunotherapy and the improvement of tumor treatment.

\section{Tumor drug resistance}

Targeting tumor cell molecular pathways is the way we treat various cancers, but tumors inevitably develop drug resistance [35]. Once the tumor becomes resistant, the side effects of the treatment increase while the effectiveness of the drug decreases significantly.

\section{The tumors of digestive system}

Studies have demonstrated that mitochondrial uncoupling protein 2 (UCP2) leads to tumor resistance to multiple anticancer drugs by reducing ROS generated by mitochondrial metabolism. Dando et al. [36] proved that the combination of genipin and everolimus could synergistically inhibit the growth of pancreatic adenocarcinoma (PAAD) cells and induce autophagy of tumor cells. This is because inhibition of UCP2 in PADD cells activates the Akt/mTOR pathway by a ROS dependent mechanism, which reduces the anti-proliferation effect of UCP2 inhibitor genipin. The hedgehog pathway mediated by zinc finger protein GLI1 plays a major role in GC. However, Yao et al. [37] demonstrated that the AKT/ mTOR pathway can activate GLI1. Moreover, the GLI1 and p-AKT expressions were correlated with tumor cell metastasis and drug resistance, and the expression level was negatively correlated with the prognosis of patients with GC.

\section{Respiratory tumors}

The wee1 inhibitor AZD1775, which targets DNA repair and cell cycle checkpoints, has been shown to be effective in some lung cancer patients, but drug resistance is also common [38, 39]. In this regard, Sen et al. [40] found that AXL receptor tyrosine kinase (ARK) expression in AZD1775-resistant small cell lung cancer (SCLC) was up-regulated. ARK can directly or through mTOR activate the extracellular regulated protein kinases (ERK) pathway to recruit and activate checkpoint kinase 1 (CHK1). These results suggest that ARK can enhance DNA damage repair by activating CHK1, which ultimately invalidates the Wee1 inhibitor. Similarly, Ye et al. [41] demonstrated that in non-small cell lung cancer (NSCLC), transmembrane-4 L-six family member-1 (TM4SF1) regulates tumor sensitivity to chemotherapy drugs by regulating the expression of discoid domain receptor 1 (DDR1)/Akt/ERK/mTOR pathway.

\section{Kidney cancer and skin cancer}

Tyrosine kinase inhibitors (TKI) inhibitors can be used to treat renal cell carcinoma (RCC), but some RCC patients 
will develop drug resistance [42]. Ishibashi et al. [43] reported that the combined treatment of interleukin-6 receptor (IL-6R) inhibitor and low-dose TKI inhibitor was more effective in inhibiting RCC growth and angiogenesis in vivo compared with the use of TKI inhibitor alone. This is because low doses of TKI inhibitors induce high levels of IL-6, which activates the AKT/mTOR pathway. These results suggest that the mechanism of failure of TKI inhibitors in some RCC patients is related to elevated IL- 6 activation of the mTOR signaling pathway.

B-Raf proto-oncogene (BRAF) mutations occur in nonmelanoma skin cancer (NMSC), but BRAF inhibitors have not been used in such tumors. The main reason is that BRAF mutated NMSC has primary or secondary resistance to BRAF inhibitors. Sen et al. [44] found a strong correlation between PI3K/mTOR signaling pathway and BRAF inhibitor resistance. This suggests that activation of the mTOR pathway may lead to BRAF mutated NMSC resistance to BRAF inhibitors. In addition, Obenauf et al. [45] found that BRAF, anaplastic receptor tyrosine kinase (ALK) and epidermal growth factor receptor (EGFR) inhibitors induced some secretion signals in cancer cells, which led to the proliferation and metastasis of drug-resistant tumor cells. Further studies have found that in melanoma cells treated with BRAF inhibitor, the downregulation of transcription factor FOS related antigen-1 (FRA1) activates multiple signaling pathways, among which the PI3K/AKT/mTOR pathway plays a major role. When the BRAF and PI3K/ AKT/mTOR signaling pathways are simultaneously inhibited, the growth of drug-resistant BRAF mutated human melanoma cells can be inhibited.

The above studies suggest that tumor cells can evade anti-tumor drug-induced cell death by activating the intracellular mTOR signaling pathway, so the activation of the mTOR signaling pathway may be one of the mechanisms of drug resistance in tumors. Drug combinations targeting the mTOR signaling pathway may be used to treat tumors that have developed resistance.

\section{Autophagy and apoptosis of cancer}

On the one hand, autophagy can keep the genome stable by removing damaged organelles and misfolded proteins, so it can inhibit the growth of cancerous cells [46]. On the other hand, autophagy provides the tumor with more nutrients, which strengthens the tumor's ability to cope with extreme environments $[47,48]$. In addition, the unlimited proliferation of tumors is partly due to the inhibition of tumor cell apoptosis.

Sun et al. [49] concluded that mTOR inhibits the expression of glycogen synthase kinase-3 (GSK-3) in prostate cancer cells. The down-regulation of GSK-3 will inhibit the caspase- 3 signaling pathway, leading to the reduction of ROS production. Decreased ROS inhibits apoptosis of tumor cells to protect prostate cancer cells. However, Zou et al. [50] reported that mTORC2 prevented cancerous inhibitor of protein phosphatase $2 \mathrm{~A}$ (CIP2A) from binding to protein phosphatase 2A (PP2A) to restore PP2A activity. PP2A reduces the transcription of miR-9-3p and upregulates the expression of E2F transcription factor 1 (E2F1) by promoting the degradation of c-Myc, so it inhibits the apoptosis of tumor cells. In addition, Yang et al. [51] reported that the expression of p-AKT, p-mTOR, P62 and B-cell lymphoma-2 (BCL-2) was significantly decreased in oral squamous cell carcinoma (OSCC) cells with long non-coding RNA (lncRNA) CASC9 knockdown, while the expression of BCL2 associated X (BAX) was increased. These results suggest that lncRNA CASC9 inhibits autophagy-mediated apoptosis through the AKT/mTOR pathway, which promotes OSCC cell proliferation.

In summary, the above studies indicate that mTOR signaling pathway can promote the occurrence and progression of tumors by regulating autophagy and apoptosis of tumor cells. In addition, the above research also provides theoretical support for clinical research on anticancer targeted drugs, which is of great significance.

\section{The research progress of $m$ TOR inhibitors in tumors New mTOR inhibitors}

Because the mTOR signaling pathway is active in most human cancers, scientists have developed first and second-generation mTOR inhibitors for cancer treatment. The U.S. food and drug administration approved temsirolimus and everolimus for use in kidney or breast cancer. Temsirolimus is used to treat advanced RCC, while everolimus is used to treat RCC that has failed to respond to sunitinib or sorafenib, in combination with exemestane for breast cancer with hormone receptor $(+)$ and Her2 (-), pancreatic neuromedicinal tumors (pNET) and subependymal giants-cell astrocytoma (SEGA).

Rodrik-Outmezguine et al. [52] connected the first and second-generation of mTOR inhibitors together to form the third generation of mTOR inhibitor rapalink-1, which can simultaneously target two targets on the mTOR enzyme. Rapalink-1, with its potent anticancer activity, reduces the size of tumors resistant to first- or secondgeneration mTOR inhibitors. This method provides a new idea and model for the design of new anticancer, antiviral and antibacterial drugs. In addition to common clinical mTOR inhibitors, a variety of drugs have been found to inhibit mTOR activity. Nguyen et al. [53] explored that the Halitulin analog ICSN3250 is a specific mTOR inhibitor, which can compete and replace phospholipids acid in the FRB domain of mTOR. Previous studies have shown that the newly discovered drug LY3023414 inhibits the 
$\mathrm{PI} 3 \mathrm{~K} / \mathrm{mTOR} / \mathrm{DNA}$ dependent protein kinase (DNA-PK) pathway in vitro tumor cells and has anti-tumor proliferation activity. Bendell et al. [54] conducted clinical trials and concluded that LY3023414 achieved sustained partial remission in endometrial cancer patients with phosphoinositide-3-kinase regulatory subunit 1 (PIK3R1) and phosphate and PTEN mutations. In addition, Plews et al. [55] proved that OSU-53 could inhibit the growth of thyroid cancer cells in vitro by activating AMPK to inhibit the mTOR pathway or directly inhibit the mTOR pathway.

These results suggest that a variety of newly discovered mTOR inhibitors are effective in many tumors. In the future, these drugs can be perfected for clinical research, so that they can be used for clinical treatment.

\section{New progress in $\mathrm{mTOR}$ inhibitors}

With the development of the mTOR pathway, many scientists have studied the effects of mTOR inhibitors in different tumors [48, 56-58]. Because conventional platinum chemotherapy failed to respond to ovarian clear cell carcinoma (OCCC), Caumanns et al. [59] conducted drug testing of mtorc1/2 inhibitor AZ D8055 in the OCCC cell lines. The results showed that the OCCC cell line was sensitive to AZD8055, and AZD8055 was validated in a xenotransplantation model. Similarly, Hanna et al. [60] demonstrated that everolimus was effective in undifferentiated thyroid cancer patients with PI3K/ mTOR/Akt mutations. Morran et al. [61] reported that rapamycin effectively blocked the proliferation and development of pancreatic cancer cells in mice with Kirsten rat sarcoma viral oncogene (KRAS) activation and PTEN mutation. Moreover, pancreatic cancer with Kirsten rat sarcoma viral oncogene (KRAS) activation and p53 mutation did not respond to rapamycin. The mTOR inhibitor temsirolimus can down-regulate the expression of tumor hypoxia-inducing factor and block the cell cycle in G1 phase to inhibit the growth of cancer cells and tumor angiogenesis. Korfel et al. [62] explored that temsirolimus can effectively treat primary central nervous system lymphoma (PCNSL), but the response was relatively short. In addition to the above effects, scientists also found that the mTOR inhibitor has a protective effect. Functional pNET can secrete 5-hydroxytryptamine (5-HT) into the blood, and over time high 5-HT in the blood can damage the heart. Orr-Asman et al. [63] found that mTOR kinase inhibitor CC-223 reduced myocardial damage by reducing cardiac valve fibrosis compared with pNET mice treated with either placebo or rapalog alone. However, Goldman et al. [64] reported that mTOR inhibitors could reduce infertility caused by ovarian damage due to chemotherapy. Table 1 summarizes the research progress of mTOR inhibitors.

\section{TSC mutations and the effects of mTOR inhibitors}

TSC1/2 is upstream of mTOR and regulates mTOR activity through Rheb. Some studies have shown that in some tumors, patients with TSC mutations are unusually sensitive to mTOR inhibitors. Wang et al. [65] found in a case of renal angiomyolipoma that somatic cell mutations in the patient stopped the translation of TSC2, which led to the activation of mTORC1, and the application of everolimus effectively reduced tumor growth and distal metastasis. Similarly, TSC mutations in RCC, chromophobe RCC, and metastatic RCC patients were reported unusually sensitive to mTOR inhibitors [66-69]. Furthermore, metastatic RCC patients with TSC mutation benefit from rapalogs are more common than the advanced patients [70]. In addition to renal tumors, hepatitis B virus (HBV)associated HCC with TSC mutations had a similar effect [71]. Levine et al. [72] analyzed data from patients with endometrioid adenocarcinoma and found that patients with TSC2 mutations had longer progression-free survival than patients with other drugs. This phenomenon

Table 1 Summary of the research phase of the mTOR inhibitors

\begin{tabular}{llll}
\hline mTOR inhibitors & Applied tumor & Phase & References \\
\hline Everolimus & RCC & FDA approved & - \\
Temsirolimus & Advanced RCC & FDA approved & - \\
ICSN3250 & Colon cancer cell & Pre-clinical studies & Nguyen et al. [53] \\
LY3023414 & Solid tumor or lymphoma & Phase I clinical trial & Bendell et al. [54] \\
OSU-53 & Thyroid cancer cell & Pre-clinical studies & Plews et al. [55] \\
AZD8055 & OCCC cell & Pre-clinical studies & Caumanns et al. [59] \\
Everolimus & Aggressive and RAIR thyroid cancer & Phase II clinical trial & Hanna et al. [60] \\
Rapamycin & Pancreatic cancer & Pre-clinical studies & Morran et al. [61] \\
Temsirolimus & PCNSL & Phase II clinical trial & Korfel et al. [62] \\
\hline
\end{tabular}

$R C C$ renal cell carcinoma, OCCC ovarian clear cell carcinoma, RAIR radioactive iodine-refractory, PCNSL primary central nervous system lymphoma, FDA Food and Drug Administration 
may be due to the abnormal activation of mTORC1 by the TSC mutation, which makes such somatic mutant patients unusually sensitive to mTOR inhibitors. Therefore, the TCS/mTOR pathway gene sequencing in tumor patients can be used to guide clinical drug therapy.

\section{Combination therapy with mTOR inhibitors}

There are feedback mechanisms in the PI3K/AKT signaling pathway, including positive and negative feedback. In the negative feedback mechanism, when the PI3K/ AKT/mTORC1 pathway is activated, the downstream S6K1 is activated. Activated S6K1 phosphorylates downstream insulin receptor substrate (IRS) so that the IRS cannot activate PI3K. This reduces the phosphorylation of phosphatidylinositol $(4,5)$-bisphosphate (PIP2) to phosphatidylinositol $(3,4,5)$-trisphosphate (PIP3), which ultimately inhibits AKT activity. In the positive feedback mechanism, activation of the PI3K/AKT pathway also activates the downstream inhibitor of nuclear factor kappa-B kinase (IKK)/nuclear factor kappa-B (NF-kB) pathway. Activated NF-B inhibits PTEN expression to reduce the dephosphorylation of PIP3, which leads to the accumulation of PIP3 and continued activation of AKT. When mTOR inhibitor inhibits mTORC1, it will inhibit the inhibitory effect of S6K1 on IRS, thus enhancing the activation of the PI3K/AKT signaling pathway [73]. Thus, although the mTOR pathway is involved in almost every process of cancer, including cell growth, proliferation, metabolism, and immune response, the activation of AKT by mTOR inhibitors limits its clinical effectiveness.

However, many studies have shown that other antitumor drugs combined with mTOR inhibitors can overcome this resistance [74]. Motzer et al. [75] demonstrated that the combination therapy of Vascular endothelial growth factor (VEGF) inhibitor lenvatinib and everolimus had good efficacy in advanced or metastatic RCC. In head and neck squamous cell carcinoma (HNSCC), MAPK/ERK kinase (MEK)/ERK/activator protein 1 (AP-1), NF- $\mathrm{kB}$ and mTOR signaling pathways are frequently activated. Mohan et al. [76] showed that PI3K/ mTOR inhibitor PF-384 could not completely inhibit the growth of xenograft HNSCC. While the combined use of PF-384 and the MEK inhibitor PD-901 can inhibit the production of IL- 8 and VEGF and the reverse activation of downstream NF- $k$ B and AP-1, thus significantly inhibiting tumor growth, proliferation, anti-apoptosis, and angiogenesis. Zhang et al. [77] concluded that inhibition of androgen receptor (AR) in HCC could feedback to activate the AKT/mTOR signaling pathway, while mTOR reduced AR degradation and promoted the expression of nuclear AR protein. In addition, Chen et al. [78] found in breast cancer that mTOR inhibitor AZD8055 can prevent the heat shock protein (HSP) 90 inhibitor AUY922 from up-regulating the response of HSP70 and HSP27 in breast cancer, while HSP90 inhibitor can block the activation of PI3K/Akt caused by mTOR inhibitor. Therefore, mTOR inhibitors can synergistically inhibit tumor growth in combination with HSP90 inhibitors or AR inhibitors.

mTOR inhibitor combination therapy has also shown activity in many types of lung cancer. Christopoulos et al. [79] reported that carboplatin and paclitaxel combined with everolimus were effective and well-tolerated in patients with metastatic large-cell neuroendocrine carcinoma (LCNEC). In addition, Hai et al. [80] showed that the combined use of mTOR and Wee1 inhibitors could synergically inhibit KRAS mutated NSCLC cell lines. This is because mTOR inhibitors inhibit DNA repair by reducing cyclin D1, which enhances the inhibition effect of Wee1 by enhancing DNA damage in NSCLC cells.

These studies suggest that a combination of mTOR inhibitors may enhance the activity of a variety of antitumor drugs, compared with drugs taken alone (Table 2). These studies guide the clinical use of mTOR inhibitors and other anti-tumor drugs.

\section{Conclusion and prospect}

The mTOR signaling pathway is closely related to tumors, and it is closely related to its cell growth, metabolism, apoptosis and autophagy [47, 81]. For example, the mTOR signaling pathway can affect gene transcription and protein synthesis to regulate cell growth and proliferation, affect the immune cell differentiation to participate in immune regulation, and play an important role in tumor metabolism. A number of studies have shown that a variety of new mTOR inhibitors show high anti-tumor activity in clinical studies, and the use of mTOR inhibitors in combination with other anti-tumor drugs has a significant effect. This review describes the relationship between the mTOR signaling pathway and tumor cell growth, metabolism, apoptosis and autophagy). This review also introduces the role of mTOR signaling in tumors of various organs and the research progress on the application of mTOR inhibitors in tumors, which indicates the importance of the mTOR signaling pathway in the tumor fields.

However, the role of the mTOR signaling pathway has not been clearly studied, and although mTOR inhibitors can inhibit tumor cell growth, their ability to induce tumor cell death is limited. The mechanisms of tumors are complex and involve many signaling pathways. Inhibition of certain signaling pathways may lead to feedback activation of other signaling pathways, so although mTOR inhibitor combination therapy is more effective, its effect is limited. In addition, clinical trials have demonstrated that the side effects of treatment with mTOR 
Table 2 Summary of mTOR inhibitors in combination with other antitumor drugs

\begin{tabular}{|c|c|c|c|c|}
\hline mTOR inhibitors & Combined drug & Applied tumor & The effect & References \\
\hline Everolimus & VEGF inhibitor lenvatinib & $\mathrm{RCC}$ & $\begin{array}{l}\text { Progression-free survival is significantly extended compared } \\
\text { to using them separately }\end{array}$ & Motzer et al. [75] \\
\hline PF-384 & MEK inhibitor PD-901 & HNSCC & $\begin{array}{l}\text { They inhibit the production of IL-8 and VEGF and the activa- } \\
\text { tion of NF-KB and AP-1 }\end{array}$ & Mohan et al. [76] \\
\hline AZD8055 & HSP90 inhibitor AUY922 & Breast cancer & $\begin{array}{l}\text { AZD } 8055 \text { inhibits the upregulation of HSP70 and HSP27 } \\
\text { induced by AUY922, while AUY922 blocks the activation of } \\
\text { PI3KVAkt induced by AZD805 }\end{array}$ & Chen et al. [78] \\
\hline Rapamycin & AR inhibitor enzalutamide & $\mathrm{HCC}$ & $\begin{array}{l}\text { Rapamycin inhibits the AKT/mTOR signaling pathway acti- } \\
\text { vated by enzalutamide, while enzalutamide inhibits the } \\
\text { up-regulation of AR expression caused by rapamycin }\end{array}$ & Zhang et al. [77] \\
\hline Everolimus & Carboplatin and paclitaxel & LCNEC & $\begin{array}{l}\text { They improve the overall response rate and disease control } \\
\text { rate }\end{array}$ & Christopoulos et al. [79] \\
\hline AZD2014 & Wee1 inhibitor AZD1775 & NSCLC & $\begin{array}{l}\text { AZD2014 enhances the effect of AZD1775 by reducing } \\
\text { cyclin D1 to enhance DNA damage }\end{array}$ & Hai et al. [80] \\
\hline
\end{tabular}

AP-1 activator protein 1, AR androgen receptor, HCC hepatic cell carcinoma, HNSCC head and neck squamous cell carcinoma, HSP90 heat shock protein $90, I L-8$ interleukin-8, LCNEC large-cell neuroendocrine carcinoma, MEK MAPK/ERK kinase, NSCLC non-small cell lung cancer, $R C C$ renal cell carcinoma, VEGF vascular growth factor

inhibitors cannot be ignored. It is hoped that the action mechanisms of the mTOR signaling pathway can be clearly studied in the future, and then selective mTOR inhibitors can be developed to improve anti-tumor activity and reduce side effects.

\section{Abbreviations}

5-HT: 5-Hydroxytryptamine; A2aR: A2a receptor; AKT: Protein kinase B; ALK: Anaplastic receptor tyrosine kinase; AMPK: Adenosine 5'-monophosphateactivated protein kinase; AP-1: Activator protein 1; AR: Androgen receptor; ARK: AXL receptor tyrosine kinase; BCAA: Branched chain amino acid; BCL-2: B-cell lymphoma-2; BMM: Bone marrow mononuclear; BRAF: B-Raf protooncogene; CCSC: Colon cancer stem cells; CD: Cluster of differentiation; CHK1: Checkpoint kinase 1; CIP2A: Cancerous inhibitor of protein phosphatase 2A; DC: Dendritic cell; DDR1: Discoid domain receptor 1; DNA-PK: DNA dependent protein kinase; E2F1: E2F transcription factor 1; EGFR: Epidermal growth factor receptor; ERa: Estrogen receptor a; ERK: Extracellular regulated protein kinases; FA: Fatty acid; FASN: Fatty acid synthase; FFAs: Free fatty acids; FRA1: FOS related antigen-1; GC: Gastric cancer; GSK-3: Glycogen synthase kinase-3; H2AX: H2A histone family member X; HBV: Hepatitis B virus; HCC: Hepatic cell carcinoma; HIF-1a: Hypoxia-inducible factor-1a; HNSCC: Head and neck squamous cell carcinoma; HSP: Heat shock protein; IKK: Inhibitor of nuclear factor kappa-B kinase; IL: Interleukin; IL-6R: Interleukin-6 receptor; IRS: Insulin receptor substrate; KRAS: Kirsten rat sarcoma viral oncogene; LCNEC: Large-cell neuroendocrine carcinoma; LKB1: Liver kinase B1; IncRNA: Long non-coding RNA; MAPKAP1: Mitogen-activated protein kinase associated protein 1; MEK: MAPK/ERK kinase; miRNA: microRNA; MMP-9: Matrix metallopeptidase 9; mTOR: Mammalian target of rapamycin; mTORC1: Mammalian target of rapamycin complex 1; mTORC2: Mammalian target of rapamycin complex 2; NF-KB: Nuclear factor kappa-B; NK: Natural killer; NMSC: Nonmelanoma skin cancer; NSCLC: Non-small cell lung cancer; OCCC: Ovarian clear cell carcinoma; OSCC: Oral squamous cell carcinoma; OTUD7B: OTU deubiquitinase 7B; PAAD: Pancreatic adenocarcinoma; PCNSL: Primary central nervous system lymphoma; PI3K: Phosphoinositide-3-kinase; PIK3R1: Phosphoinositide-3-kinase regulatory subunit 1; PIP2: Phosphatidylinositol (4,5)-bisphosphate; PIP3: Phosphatidylinositol $(3,4,5)$-trisphosphate; PK: Pyruvate kinase; PKM2: Pyruvate kinase M2; pNET: Pancreatic neuroendocrine tumors; PP2A: Protein phosphatase 2A; PTEN: Phosphate and tension homology deleted on chromosome ten; RCC: Renal cell carcinoma; RNF168: Ring finger protein 168; ROS: Reactive oxygen species; SCLC: Small cell lung cancer; SEGA: Subependymal giantscell astrocytoma; SLC7A5: Solute carrier family 7 member 5; SREBP: Sterol regulatory element binding protein; STAT5: Signal transducer and activator of transcription 5; TFE: Transcription factor enhancer; TKI:Tyrosine kinase inhibitors; TM4SF1: Transmembrane-4 L-six family member-1; TRAF2: TNF receptor associated factor 2; TSC1: Tuberous sclerosis complex subunit 1;TSC2: Tuberous sclerosis complex subunit 2; UCP2: Uncoupling protein 2; VEGF: Vascular endothelial growth factor.

\section{Acknowledgements}

We would like to thank Alan Larson (School of Medicine, University of California San Diego) for English language revision.

\section{Authors' contributions}

All authors revised the final manuscript. All authors read and approved the final manuscript.

\section{Funding}

This work was supported partly by National Natural Science Foundation of China (81541153); Guangdong Provincial Science and Technology Department (2016A050503046, 2015A050502048 and 2016B030309002); Southern Marine Science and Engineering Guangdong Laboratory Zhanjiang (ZJW2019-07): The Public Service Platform of South China Sea for R\&D Marine Biomedicine Resources (GDMUK201808); Natural Science Cultivation Project of Guangdong Medical University (GDMUM201811); and "Group-type" Special Supporting Project for Educational Talents in Universities (4SG19057G); Zhanjiang Science and Technology Plan (2017A06012).

\section{Availability of data and materials}

Not applicable.

\section{Ethics approval and consent to participate}

Not applicable.

\section{Consent for publication}

All authors consent for publication.

\section{Competing interests}

The authors declare that they have no competing interests.

\section{Author details}

${ }^{1}$ Guangdong Key Laboratory for Research and Development of Natural Drugs, Guangdong Medical University, Zhanjiang, China. ${ }^{2}$ Marine Medical Research Institute of Guangdong Zhanjiang (GDZJMMRI), Southern Marine Science and Engineering Guangdong Laboratory Zhanjiang, Guangdong Medical University, Zhanjiang, China. ${ }^{3}$ Department of Pathology, Guangdong Medical 
University, Dongguan, China. ${ }^{4}$ Department of Gastroenterology, Zibo Central Hospital, Zibo, China.

Received: 18 December 2019 Accepted: 26 February 2020 Published online: 10 March 2020

\section{References}

1. Unni N, Arteaga CL. Is dual mTORC1 and mTORC2 therapeutic blockade clinically feasible in cancer? JAMA Oncol. 2019. https://doi.org/10.1001/ jamaoncol.2019.2525 (Epub ahead of print)

2. Dowling RJ, Topisirovic I, Fonseca BD, Sonenberg N. Dissecting the role of mTOR: lessons from mTOR inhibitors. Biochim Biophys Acta. 2010;1804(3):433-9.

3. Neufeld TP. TOR-dependent control of autophagy: biting the hand that feeds. Curr Opin Cell Biol. 2010;22(2):157-68.

4. Zoncu R, Efeyan A, Sabatini DM. mTOR: from growth signal integration to cancer, diabetes and ageing. Nat Rev Mol Cell Biol. 2011;12(1):21-35.

5. Saxton RA, Sabatini DM. mTOR signaling in growth, metabolism, and disease. Cell. 2017;168(6):960-76.

6. Mossmann D, Park S, Hall MN. mTOR signalling and cellular metabolism are mutual determinants in cancer. Nat Rev Cancer. 2018;18(12):744-57.

7. Guo B, Li D, Du L, Zhu X. piRNAs: biogenesis and their potential roles in cancer. Cancer Metastasis Rev. 2020. https://doi.org/10.1007/s10555-02009863-0 (Epub ahead of print)

8. Hsieh AC, Liu Y, Edlind MP, Ingolia NT, Janes MR, Sher A, et al. The translational landscape of mTOR signalling steers cancer initiation and metastasis. Nature. 2012:485(7396):55-61.

9. Nathan N, Keppler-Noreuil KM, Biesecker LG, Moss J, Darling TN. Mosaic disorders of the PI3K/PTEN/AKT/TSC/mTORC1 signaling pathway. Dermatol Clin. 2017;35(1):51-60.

10. Lim HJ, Crowe P, Yang JL. Current clinical regulation of PI3K/PTEN/Akt/ mTOR signalling in treatment of human cancer. J Cancer Res Clin Oncol. 2015:141(4):671-89.

11. Hou W, Liu J, Chen P, Wang H, Ye BC, Qiang F. Mutation analysis of key genes in RAS/RAF and PI3K/PTEN pathways in Chinese patients with hepatocellular carcinoma. Oncol Lett. 2014;8(3):1249-54.

12. Zhang Y, Zhang J, Xu K, Xiao Z, Sun J, Xu J, et al. PTEN/PI3K/mTOR/B7-H1 signaling pathway regulates cell progression and immuno-resistance in pancreatic cancer. Hepatogastroenterology. 2013;60(127):1766-72.

13. Chen JS, Wang Q, Fu XH, Huang XH, Chen XL, Cao LQ, et al. Involvement of PI3K/PTEN/AKT/mTOR pathway in invasion and metastasis in hepatocellular carcinoma: association with MMP-9. Hepatol Res. 2009;39(2):177-86.

14. Chen S, Fisher RC, Signs S, Molina LA, Shenoy AK, Lopez MC, et al. Inhibition of PI3K/Akt/mTOR signaling in PI3KR2-overexpressing colon cancer stem cells reduces tumor growth due to apoptosis. Oncotarget. 2017;8(31):50476-88

15. Xie X, Hu H, Tong X, Li L, Liu X, Chen M, et al. The mTOR-S6K pathway links growth signalling to DNA damage response by targeting RNF168. Nat Cell Biol. 2018;20(3):320-31.

16. Deng $L$, Chen $L$, Zhao L, Xu Y, Peng $X$, Wang $X$, et al. Ubiquitination of Rheb governs growth factor-induced mTORC1 activation. Cell Res. 2019;29(2):136-50.

17. Wang B, Jie Z, Joo D, Ordureau A, Liu P, Gan W, et al. TRAF2 and OTUD7B govern a ubiquitin-dependent switch that regulates mTORC2 signalling Nature. 2017;545(7654):365-9.

18. Kovalski JR, Bhaduri A, Zehnder AM, Neela PH, Che Y, Wozniak GG, et al. The functional proximal proteome of oncogenic Ras includes mTORC2. Mol Cell. 2019;73(4):830-44.e12.

19. Liu J, Li D, Luo H, Zhu X. Circular RNAs: the star molecules in cancer. Mol Aspects Med. 2019;70:141-52.

20. Ricoult SJ, Yecies JL, Ben-Sahra I, Manning BD. Oncogenic PI3K and K-Ras stimulate de novo lipid synthesis through mTORC1 and SREBP. Oncogene. 2016;35(10):1250-60.

21. Tao T, Su Q, Xu S, Deng J, Zhou S, Zhuang Y, et al. Down-regulation of PKM2 decreases FASN expression in bladder cancer cells through AKT/ mTOR/SREBP-1c axis. J Cell Physiol. 2019;234(3):3088-104.
22. Di Malta C, Siciliano D, Calcagni A, Monfregola J, Punzi S, Pastore N, et al. Transcriptional activation of RagD GTPase controls mTORC1 and promotes cancer growth. Science. 2017;356(6343):1188-92.

23. Guri Y, Colombi M, Dazert E, Hindupur SK, Roszik J, Moes S, et al. mTORC2 promotes tumorigenesis via lipid synthesis. Cancer Cell. 2017:32(6):80723.e12.

24. Zhu X, Zhang J, Fan W, Wang F, Yao H, Wang Z, et al. The rs391957 variant cis-regulating oncogene GRP78 expression contributes to the risk of hepatocellular carcinoma. Carcinogenesis. 2013;34(6):1273-80.

25. Ericksen RE, Lim SL, McDonnell E, Shuen WH, Vadiveloo M, White PJ, et al. Loss of BCAA catabolism during carcinogenesis enhances mTORC1 activity and promotes tumor development and progression. Cell Metab. 2019;29(5):1151-65.e6.

26. Shi L, Wu Z, Miao J, Du S, Ai S, Xu E, et al. Adenosine interaction with adenosine receptor $\mathrm{A} 2 \mathrm{a}$ promotes gastric cancer metastasis by enhancing PI3K-AKT-mTOR Signaling. Mol Biol Cell. 2019. https://doi. org/10.1091/mbc.E19030136.

27. Madak-Erdogan Z, Band S, Zhao YC, Smith BP, Kulkoyluoglu-Cotul E, Zuo $Q$, et al. Free fatty acids rewire cancer metabolism in obesity-associated breast cancer via estrogen receptor and mTOR signaling. Cancer Res. 2019;79(10):2494-510.

28. Tan S, Li D, Zhu X. Cancer immunotherapy: Pros, cons and beyond. Biomed Pharmacother. 2020;124:109821.

29. Yang K, Blanco DB, Chen X, Dash P, Neale G, Rosencrance C, et al. Metabolic signaling directs the reciprocal lineage decisions of alphabeta and gammadelta T cells. Sci Immunol. 2018. https://doi.org/10.1126/sciim munol.aas9818.

30. Essig K, Hu D, Guimaraes JC, Alterauge D, Edelmann S, Raj T, et al. Roquin suppresses the PI3K-mTOR signaling pathway to inhibit T helper cell differentiation and conversion of Treg to Tfr Cells. Immunity. 2017:47(6):1067-82.e12.

31. Pollizzi KN, Patel $\mathrm{CH}$, Sun $\mathrm{H}$, Oh MH, Waickman AT, Wen J, et al. mTORC1 and $\mathrm{mTORC} 2$ selectively regulate CD8(+) T cell differentiation. J Clin Invest. 2015;125(5):2090-108.

32. Wang $F$, Meng M, Mo B, Yang Y, Ji Y, Huang P, et al. Crosstalks between mTORC1 and mTORC2 variagate cytokine signaling to control NK maturation and effector function. Nat Commun. 2018;9(1):4874.

33. Chen YL, Lin HW, Sun NY, Yie JC, Hung HC, Chen CA, et al. mTOR inhibitors can enhance the anti-tumor effects of DNA vaccines through modulating dendritic cell function in the tumor microenvironment. Cancers. 2019;11(5):617.

34. Zhihua Y, Yulin T, Yibo W, Wei D, Yin C, Jiahao X, et al. Hypoxia decreases macrophage glycolysis and M1 percentage by targeting microRNA-30c and mTOR in human gastric cancer. Cancer Sci. 2019;110(8):2368-77.

35. Zhu X, Lin MCM, Fan W, Tian L, Wang J, Ng SS, et al. An intronic polymorphism in GRP78 improves chemotherapeutic prediction in non-small cell lung cancer. Chest. 2012;141(6):1466-72.

36. Dando I, Pacchiana R, Pozza ED, Cataldo I, Bruno S, Conti P, et al. UCP2 inhibition induces ROS/Akt/mTOR axis: role of GAPDH nuclear translocation in genipin/everolimus anticancer synergism. Free Radic Biol Med. 2017:113:176-89.

37. Yao Y, Zhou D, Shi D, Zhang H, Zhan S, Shao X, et al. GLI1 overexpression promotes gastric cancer cell proliferation and migration and induces drug resistance by combining with the AKT-mTOR pathway. Biomed Pharmacother. 2019;111:993-1004.

38. Zhu X, Luo H, Xu Y. Transcriptome analysis reveals an important candidate gene involved in both nodal metastasis and prognosis in lung adenocarcinoma. Cell Biosci. 2019:9:92.

39. Zhu X, Gao S, Luo H, Fan W, Guo S, Yao H, et al. 9q33.3, a stress-related chromosome region, contributes to reducing lung squamous cell carcinoma risk. J Thorac Oncol. 2014;9(7):1041-7.

40. Sen T, Tong P, Diao L, Li L, Fan Y, Hoff J, et al. Targeting AXL and mTOR pathway overcomes primary and acquired resistance to WEE1 inhibition in small-cell lung cancer. Clin Cancer Res. 2017:23(20):6239-53.

41. Ye L, Pu C, Tang J, Wang Y, Wang C, Qiu Z, et al. Transmembrane-4 L-six family member-1 (TM4SF1) promotes non-small cell lung cancer proliferation, invasion and chemo-resistance through regulating the DDR1/ Akt/ERK-mTOR axis. Respir Res. 2019;20(1):106.

42. Tang Z, Li D, Hou S, Zhu X. The cancer exosomes: clinical implications, applications and challenges. Int J Cancer. 2019. https://doi.org/10.1002/ ijc.32762 (Epub ahead of print). 
43. Ishibashi K, Haber T, Breuksch I, Gebhard S, Sugino T, Kubo H, et al. Overriding TKI resistance of renal cell carcinoma by combination therapy with IL-6 receptor blockade. Oncotarget. 2017;8(33):55230-45.

44. Sen S, Meric-Bernstam F, Hong DS, Hess KR, Subbiah V. Co-occurring genomic alterations and association with progression-free survival in BRAFV600-mutated nonmelanoma tumors. J Natl Cancer Inst. 2017. https ://doi.org/10.1093/jnci/djx094

45. Obenauf AC, Zou Y, Ji AL, Vanharanta S, Shu W, Shi H, et al. Therapyinduced tumour secretomes promote resistance and tumour progression. Nature. 2015;520(7547):368-72.

46. Tang $X$, Huang $Y$, Lei J, Luo $H$, Zhu $X$. The single-cell sequencing: new developments and medical applications. Cell Biosci. 2019;9:53.

47. Zhou Y, Wang Y, Zhou W, Chen T, Wu Q, Chutturghoon VK, et al. YAP promotes multi-drug resistance and inhibits autophagy-related cell death in hepatocellular carcinoma via the RAC1-ROS-mTOR pathway. Cancer Cell Int. 2019;19:179.

48. Li K, Luo H, Huang L, Luo H, Zhu X. Microsatellite instability: a review of what the oncologist should know. Cancer Cell Int. 2020;20:16

49. Sun Y, Ai JZ, Jin X, Liu LR, Lin TH, Xu H, et al. IL-8 protects prostate cancer cells from GSK-3beta-induced oxidative stress by activating the mTOR signaling pathway. Prostate. 2019;79(10):1180-90.

50. Zou Z, Chen J, Liu A, Zhou X, Song Q, Jia C, et al. mTORC2 promotes cell survival through c-Myc-dependent up-regulation of E2F1. J Cell Biol. 2015:211(1):105-22.

51. Yang Y, Chen D, Liu H, Yang K. Increased expression of IncRNA CASC9 promotes tumor progression by suppressing autophagy-mediated cell apoptosis via the AKT/mTOR pathway in oral squamous cell carcinoma. Cell Death Dis. 2019;10(2):41.

52. Rodrik-Outmezguine VS, Okaniwa M, Yao Z, Novotny CJ, McWhirter C, Banaji A, et al. Overcoming mTOR resistance mutations with a newgeneration mTOR inhibitor. Nature. 2016;534(7606):272-6.

53. Nguyen TL, Nokin MJ, Egorov M, Tome M, Bodineau C, Di Primo C, et al. mTOR inhibition via displacement of phosphatidic acid induces enhanced cytotoxicity specifically in cancer cells. Cancer Res. 2018;78(18):5384-97.

54. Bendell JC, Varghese AM, Hyman DM, Bauer TM, Pant S, Callies S, et al. A first-in-human phase 1 study of LY3023414, an oral PI3K/mTOR dual inhibitor, in patients with advanced cancer. Clin Cancer Res. 2018;24(14):3253-62.

55. Plews RL, Mohd Yusof A, Wang C, Saji M, Zhang X, Chen CS, et al. A novel dual AMPK activator/mTOR inhibitor inhibits thyroid cancer cell growth. J Clin Endocrinol Metab. 2015:100(5):E748-E756756.

56. Piselli P, Taborelli M, Cimaglia C, Serraino D, Italian T, Cancer Cohort S. Decreased incidence of Kaposi sarcoma after kidney transplant in Italy and role of mTOR-inhibitors: 1997-2016. Int J Cancer. 2019;145(2):597-8.

57. Park YL, Kim HP, Cho YW, Min DW, Cheon SK, Lim YJ, et al. Activation of WNT/beta-catenin signaling results in resistance to a dual PI3K/mTOR inhibitor in colorectal cancer cells harboring PIK3CA mutations. Int J Cancer. 2019;144(2):389-401.

58. Tang J, Xu Z, Huang L, Luo H, Zhu X. Transcriptional regulation in model organisms: recent progress and clinical implications. Open Biol. 2019;9(11):190183.

59. Caumanns JJ, Berns K, Wisman GBA, Fehrmann RSN, Tomar T, Klip H, et al. Integrative kinome profiling identifies mTORC1/2 inhibition as treatment strategy in ovarian clear cell carcinoma. Clin Cancer Res. 2018;24(16):3928-40.

60. Hanna GJ, Busaidy NL, Chau NG, Wirth LJ, Barletta JA, Calles A, et al. Genomic correlates of response to everolimus in aggressive radioiodine-refractory thyroid cancer: a phase II study. Clin Cancer Res. 2018;24(7):1546-53.

61. Morran DC, Wu J, Jamieson NB, Mrowinska A, Kalna G, Karim SA, et al. Targeting mTOR dependency in pancreatic cancer. Gut. 2014;63(9):1481-9.

62. Korfel A, Schlegel U, Herrlinger U, Dreyling M, Schmidt C, von Baumgarten $\mathrm{L}$, et al. Phase II trial of temsirolimus for relapsed/refractory primary CNS lymphoma. J Clin Oncol. 2016;34(15):1757-63.

63. Orr-Asman MA, Chu Z, Jiang M, Worley M, LaSance K, Koch SE, et al. mTOR kinase inhibition effectively decreases progression of a subset of neuroendocrine tumors that progress on rapalog therapy and delays cardiac impairment. Mol Cancer Ther. 2017;16(11):2432-41.
64. Goldman KN, Chenette D, Arju R, Duncan FE, Keefe DL, Grifo JA, et al. mTORC1/2 inhibition preserves ovarian function and fertility during genotoxic chemotherapy. Proc Natl Acad Sci USA. 2017;114(12):3186-91.

65. Wang T, Xie S, Luo R, Shi L, Bai P, Wang X, et al. Two novel TSC2 mutations in renal epithelioid angiomyolipoma sensitive to everolimus. Cancer Biol Ther. 2020;21(1):4-11.

66. Roldan-Romero JM, Beuselinck B, Santos M, Rodriguez-Moreno JF, Lanillos J, Calsina B, et al. PTEN expression and mutations in TSC1, TSC2 and MTOR are associated with response to rapalogs in patients with renal cell carcinoma. Int J Cancer. 2020;146(5):1435-44.

67. Maroto P, Anguera G, Roldan-Romero JM, Apellaniz-Ruiz M, Algaba F, Boonman J, et al. Biallelic TSC2 mutations in a patient with chromophobe renal cell carcinoma showing extraordinary response to temsirolimus. J Natl Compr Cancer Netw. 2018;16(4):352-8.

68. Jacob LA, Shafi G. TSC1/2 mutations as markers of response to everolimus in metastatic renal cell carcinoma: a case study. Indian J Cancer. 2019;56(3):274-5

69. Liang $B$, Ding $H$, Huang L, Luo H, Zhu X. GWAS in cancer: progress and challenges. Mol Genet Genom. 2020. https://doi.org/10.1007/s0043 8-020-01647-z (Epub ahead of print)

70. Kwiatkowski DJ, Choueiri TK, Fay AP, Rini BI, Thorner AR, de Velasco G, et al. Mutations in TSC1, TSC2, and MTOR are associated with response to rapalogs in patients with metastatic renal cell carcinoma. Clin Cancer Res. 2016;22(10):2445-522.

71. Ho DWH, Chan LK, Chiu YT, Xu IMJ, Poon RTP, Cheung TT, et al. TSC1/2 mutations define a molecular subset of HCC with aggressive behaviour and treatment implication. Gut. 2017:66(8):1496-506.

72. Levine DA, Dizon DS, Carlson JW, Filiaci VL, Powell MA, Secord AA, et al. Predictive biomarkers of endometrial cancer response: results from NRG Oncology/Gynecologic Oncology Group study 86P. Gynecol Oncol. 2018;149:12-3

73. Wan X, Harkavy B, Shen N, Grohar P, Helman LJ. Rapamycin induces feedback activation of Akt signaling through an IGF-1R-dependent mechanism. Oncogene. 2007;26(13):1932-40.

74. Liang X, Li D, Leng S, Zhu X. RNA-based pharmacotherapy for tumors: from bench to clinic and back. Biomed Pharmacother. 2020;125:109997.

75. Motzer RJ, Hutson TE, Glen H, Michaelson MD, Molina A, Eisen T, et al. Lenvatinib, everolimus, and the combination in patients with metastatic renal cell carcinoma: a randomised, phase 2, open-label, multicentre trial. Lancet Oncol. 2015;16(15):1473-82.

76. Mohan S, Vander Broek R, Shah S, Eytan DF, Pierce ML, Carlson SG, et al. MEK Inhibitor PD-0325901 overcomes resistance to PI3K/mTOR inhibitor PF-5212384 and potentiates antitumor effects in human head and neck squamous cell carcinoma. Clin Cancer Res. 2015;21(17):3946-56.

77. Zhang H, Li XX, Yang Y, Zhang Y, Wang HY, Zheng XFS. Significance and mechanism of androgen receptor overexpression and androgen receptor/mechanistic target of rapamycin cross-talk in hepatocellular carcinoma. Hepatology. 2018;67(6):2271-86.

78. Chen SM, Guo CL, Shi JJ, Xu YC, Chen Y, Shen YY, et al. HSP90 inhibitor AUY922 abrogates up-regulation of RTKs by mTOR inhibitor AZD8055 and potentiates its antiproliferative activity in human breast cancer. Int J Cancer. 2014;135(10):2462-74.

79. Christopoulos P, Engel-Riedel W, Grohe C, Kropf-Sanchen C, von Pawel J, Gutz S, et al. Everolimus with paclitaxel and carboplatin as first-line treatment for metastatic large-cell neuroendocrine lung carcinoma: a multicenter phase II trial. Ann Oncol. 2017;28(8):1898-902.

80. Hai J, Liu S, Bufe L, Do K, Chen T, Wang X, et al. Synergy of WEE1 and mTOR inhibition in mutant KRAS-driven lung cancers. Clin Cancer Res. 2017;23(22):6993-7005.

81. Wang Z, Guo J, Han X, Xue M, Wang W, Mi L, et al. Metformin represses the pathophysiology of AAA by suppressing the activation of PI3K/AKT/ mTOR/autophagy pathway in ApoE(-/-) mice. Cell Biosci. 2019;9:68.

\section{Publisher's Note}

Springer Nature remains neutral with regard to jurisdictional claims in published maps and institutional affiliations. 